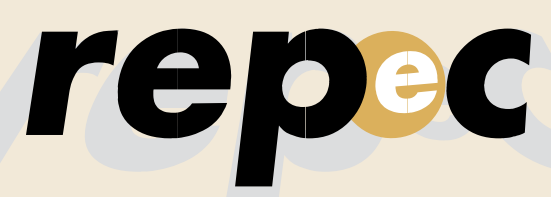

REPeC, Brasília, v. 13, n. 2, art. 2, p. 143-161, abr./jun. 2019

Disponivel online em www.repec.org.br

DOl: http://dx.doi.org/10.17524/repec.v13i2.1938
Revista de Educação e Pesquisa em Contabilidade

Journal of Education and Research in Accounting

Periódico Trimestral, digital e gratuito publicado pela Academia Brasileira de Ciências Contábeis

ISSN 1981-8610

\title{
Análise demográfica e acadêmica da autorregulação da aprendizagem de estudantes de Ciências Contábeis de IES em Salvador
}

\section{Resumo}

Objetivo: Analisar se fatores demográficos e acadêmicos influenciam a aprendizagem autorregulada.

Método: A amostra da pesquisa abrangeu um total de 713 discentes em Contabilidade de três Instituições de Ensino Superior (IES). Os dados do estudo foram coletados por meio de questionário e, para a análise estatística, foram utilizados métodos estatísticos descritivos, análises inferenciais por meio da aplicação de Análise Fatorial e Regressão Múltipla.

Resultados: Os resultados encontrados apresentaram significância para o gênero, tempo de estudo, escolaridade do pai, idade e estágio no curso de Contabilidade. Assim, estudantes do gênero feminino, jovens, que alocam mais horas aos estudos, em estágio inicial do curso e que tenha pai com baixa escolaridade tende a possuir um comportamento mais autorregulado na aprendizagem.

Contribuições: A autorregulação da aprendizagem no indivíduo pode ser influenciada por fatores sociais e demográfico, e assim é promovida à medida que é estimulada por fatores sociais externos.

Palavras-chave: Autorregulação na aprendizagem. Fatores acadêmicos. Fatores demográficos. Aprendizagem.
José Hilton Santos Aguiar

Orcid: https://orcid.org/0000-0002-2642-8342 E-mail: jhilton@ufba.br

Antonio Carlos Ribeiro da Silva Orcid: https://orcid.org/0000-0002-3255-8282 E-mail: profacr@hotmail.com 


\section{Introdução}

Com o advento das Tecnologias de Informação e Comunicação (TICs) e o aumento expressivo da quantidade de informação de fácil acesso, torna-se imperioso repensar um modelo de aprendizagem que se adéque às novas necessidades do contexto social atual, sobretudo a necessidade de aprender ou aprender a aprender. Atributos como "maior independência por parte dos aprendizes" são fundamentais para ter acesso às mais variadas fontes de informação ao compreender que, no ensino superior, por exemplo, tem-se limitação da carga horária de cada componente curricular e falta de tempo hábil por parte dis professores para esgotar todos os conteúdos.

Nesse contexto, tem-se discutido na área da Psicologia Sociocognitiva características da autorregulação em que o aprendiz se torna constructo da sua aprendizagem e é capaz de iniciar, monitorar e controlar o seu próprio modo de aprender. Zimmerma \& Martinez-Pons (1986) observaram que aqueles alunos tidos como independentes ou autorregulados possuíam atributos diferentes daqueles que apresentavam desempenho inferior, pois eram mais ativos no processo do aprender e regulavam tanto variáveis comportamentais e motivacionais quanto metacognitivas.

Para Zimmerma \& Martinez-Pons (1986) o desenvolvimento da autorregulação no indivíduo ocorre de forma gradativa até ocorrer a menor necessidade de ajuda social e envolve os níveis da observação, emulação, autocontrole e a autorregulação. Com esse preâmbulo, percebe-se que a Teoria Social Cognitiva não compreende a autorregulação de modo isolado, pois considera as características desenvolvimentais (ajuda dos pais, por exemplo) e ambientais (relativo aos fatores externos ao indivíduo: ajuda do professor, de colegas de turma, etc.) associadas à determinação de um julgamento pessoal do uso de estratégias cognitivas, metacognitivas e de autorreforçamento (Zimmerman, 2000; 2002).

Para Veiga Simão e Frison (2013), na perspectiva da teoria Sociocognitiva, da qual integra o aprendizado autorregulado, os fatores internos e externos ao indivíduo influenciam o processo da aprendizagem, uma vez que considera que a motivação, cognição e comportamento são influenciados tanto pelo indivíduo quanto pelo meio físico e social que o circunda. Assim, a autorregulação é uma interação de processos comportamentais, pessoais e ambientais (Bandura, 1986).

Portanto, a autorregulação da aprendizagem pode ser influenciada por múltiplos variáveis, seja endógenas (motivações, objetivos, etc.) ou exógenas (ajuda de pares, professores, pais, etc.) ao indivíduo. As relações de fatores exógenos ao indivíduo estão associadas à fase do processo cíclico da autorregulação e à autoeficácia (crença na capacidade de lidar e concretizar uma tarefa) (Zimmerman \& Schunk, 1989).

Com esse entendimento, a literatura nacional tem se debruçado na compreensão da influência de poucos fatores demográficos (gênero, idade) e acadêmico (semestre em curso) sobre a sobre o Self-Regulated Learning (SRL) em estudantes em Contabilidade (Silva et al., 2017; Aguiar \& Silva, 2017; Lima Filho, Lima \& Bruni, 2015). Com objetivo de compreender outros fatores que possam se relacionar com a Aprendizagem Autorregulada, este estudo busca explicar o processo da autorregulação com base no Modelo Sociocognitivo de Bandura (1986), e da teoria do Aprendizado Autorregulado, de Zimmerman e Martinez-Pons (1986), por meio do seguinte problema: Que fatores demográficos e acadêmicos influenciam na aprendizagem autorregulada de discentes em Contabilidade nas IES de Salvador (BA)?

O objetivo geral desse estudo é verificar os fatores demográficos e acadêmicos que influenciam a aprendizagem autorregulada de discentes em Contabilidade de Instituições de Ensino Superior soteropolitanas. Para atingir o objetivo geral, foram definidos os seguintes objetivos específicos: a) identificar as estratégias autorreguladas utilizadas por estudantes de graduação em Ciências Contábeis de Instituições de Ensino Superior em Salvador (BA); b) examinar as dimensões de estratégias autorreguladas utilizadas pelos estudantes de graduação em Ciências Contábeis; c) analisar se fatores demográficos e acadêmicos podem influenciar a aprendizagem autorregulada de discentes de Contabilidade nas IES de Salvador. 
Esse estudo buscou preencher lacunas do conhecimento sobre a relação de fatores que podem ser relevantes para explicar um maior grau na autorregulação na aprendizagem ao analisar outros fatores acadêmicos (tipo de IES pública ou particular) e fatores demográficos (escolaridade do pai e da mãe; atividade de trabalho ou estágio; e tempo de estudo) não analisados pela literatura brasileira. A melhor compreensão teórica de tais fatores podem ser potencializadores a ponto de torná-los mais autorregulados no processo de aprendizagem, uma vez que possibilita que comportamentos sejam modificados a fim de promover melhor desempenho acadêmico, pois o estudante autorregulado é orientado ao sucesso; e prática e social quando profissional, pois serão mais qualificados e preparados para se adequar, posicionar e retroalimentar de informações específicas para o bom funcionamento do setor e da economia (Aecc, 1990; Aicpa, 2000).

Para alcance dos objetivos pretendidos, foram aplicados questionários a uma amostra de 713 discentes de graduação em Ciências Contábeis advindos de três Instituições de Ensino Superior, localizadas no município de Salvador, Bahia. Os dados obtidos sofreram três diferentes tipos de tratamentos: estatística descritiva, para alcance do primeiro objetivo específico; análise fatorial, correspondendo ao segundo objetivo específico; e regressão múltipla, para atingir o objetivo específico três.

Os resultados apontaram que os estudantes de Contabilidade utilizam as estratégias de aprendizagem autorregulada com uma frequência superior a 68\%; o agrupamento das quatorze estratégias de autorregulação em dois grupos, pelos estudantes analisados; e a constatação da influência significativa na autorregulação da aprendizagem para as variáveis "gênero", "tempo de estudo", "idade" e "escolaridade do pai", no qual, este último fator apresentou relação negativa com o grau de autorregulação da aprendizagem, achado este não apontado pela anterior sobre a autorregulação da aprendizagem.

\section{Referêncial Teórico}

\subsection{Teoria do aprendizado autorregulado}

Estudos realizados sobre o ambiente acadêmico por Fischer, Davel e Vergara (2006) apontaram que as práticas vivenciadas e as experiências vividas e imaginárias, assim como a cognição e a emoção, são fatores que contribuem para o processo de aprendizagem. Shuell (1986) concorda que aquilo que o aprendiz faz na determinação do aprendizado é mais importante do que o professor pode proporcionar. Portanto, esses autores consideraram que processo de ensino e aprendizagem é influenciado por uma ampla variedade de características, ou seja, não é restrito apenas aos ambientes de instituições de ensino ou nas relações entre professores e alunos.

Nesse contexto que surgiu a ideia de estudar estratégias de independência utilizadas por estudantes, por volta de 1980, quando pesquisadores analisaram como os estudantes organizavam o aprendizado, e posteriormente culminou na sistematização da teoria da Aprendizagem Autorregulada ou Self-Regulated Learn (SRL). Segundo essa teoria, um estudante tido como 'autorregulado' é capaz de iniciar, monitorar e controlar o seu aprendizado (Boekaerts \& Cascallar, 2006; Efklides, 2011; Zimmerman, 2000). Dessa forma, o estudante é ativo no processo de aprendizagem, controla seus comportamentos, motivações e a metacognização com objetivo de garantir a aprendizagem até que se consiga o sucesso.

Na perspectiva da concepção sociocognitiva, a aprendizagem autorregulada ou Self-Regulated Learner (SRL) é compreendida como um processo voluntário e consciente que permite gerir o comportamento, sentimento e pensamento no intuito de alcance de metas pessoais do indivíduo, guiada por um padrão de comportamento em que o sujeito se automonitora, autoavalia e autoreage (Bandura, 1991; Polydoro \& Azzi, 2008 e Zimmerman, 2000b). Ou seja, o processo de autorregulação da aprendizagem caracteriza-se por envolver da mobilização de operações mentais ao planejamento, verificação da condução e avaliação em torno de um objetivo estabelecido por um indivíduo. 
As competências de autorregulação do aluno se desenvolve, incialmente, por influências sociais e, posteriormente, passam a ser controladas pelo próprio indivíduo e indicam quatro níveis referentes a esse processo (Schunk \& Zimmerman,1997). No primeiro nível, o aprendente tem o conhecimento sobre as habilidades de aprendizagem, que é aprendida por meio da descrição verbal ou da observação repetida. $\mathrm{O}$ estudante aprende as características mais significativas das estratégias com auxílio da modelagem social. Como exemplo, professores que apresentam um lançamento contábil e acompanham como implementar uma estratégia, habilidade ou processo de forma verbal.

No nível dois, emulativo, o estudante repete os estilos do modelo, que é considerado como padrão, em maior proporção do que copiar suas ações. Nessa fase guiada, o professor escolhe uma atividade ou estratégia e considera que os estudantes já conhecem as estratégias e tem a necessidade de colocá-las em prática; por fim, recebe um feedback da execução do processo para desenvolver as suas capacidades.

No nível três, autocontrolado, o estudante executa outra atividade que seja idêntica às anteriores que serviram de alicerce para desenvolvimento da estratégia de aprendizagem, chamado de "transferência”. A representação interna que o estudante já possui, permite a internalização da estratégia, ainda que se considere a forte dependência com o modelo-padrão trabalhado nas etapas anteriores. Após concluído, o estudante define seu autorreforço como resultado de sucesso do seu desempenho e se autoavalia. $\mathrm{O}$ momento de autoavaliação é essencial a presença do professor (Rosário, 2004).

A quarta e última etapa é a autorregulação. Os estudantes nesse nível, conseguem manter a motivação em torno dos seus objetivos a serem alcançados, assim como na sua crença da própria capacidade para aprender ou possuir melhor desempenho (autoeficácia) (Bandura, 1986; Schunk \& Zimmerman, 1997). Essa etapa também considera que os estudantes já possuem autonomia para decidir quando utilizar as estratégias, com ou sem a condução de modelos, pois já estão aptos a regular suas cognições, comportamentos e motivações, ou seja, ser autorregulado.

Com esse entendimento, os aspectos que permeiam a discussão da autorregulação na aprendizagem envolvem a interferência de características que não são exclusivamente próprias ao indivíduo, mas que podem influenciar o seu comportamento no sentido de potencializar, ou não, a autorregulação na aprendizagem.

Estudos de Corrêa-Vieira, Bragagnolo-Frison e Veiga-Simão (2015) verificaram se os alunos de uma turma de $8^{\text {a }}$ série do ensino fundamental de uma escola pública brasileira melhoraram a compreensão leitora e as competências de autorregulação da leitura ao participarem de uma intervenção pedagógica de modelagem metacognitiva. Os resultados ainda apontaram que 70,37\% dos alunos apresentaram progressos na compreensão leitora e que as estratégias autorregulatórias de compreensão leitora auxiliaram tanto na avaliação do processo de ler como na compreensão propriamente dita, ou seja, no entendimento que o estudante teve ao ler um texto.

Estudos realizados por Herndon e Bembenutty (2017), nos Estados Unidos, verificaram como fatores sociais e acadêmicos podem se relacionar com o desempenho acadêmico e constatou que as horas de estudo, autorregulação, motivação intrínseca, crenças de autoeficácia, atraso acadêmico de gratificação e nível de escolaridade foram relacionadas ao desempenho acadêmico. Esses achados sugerem que há diferenças individuais nas maneiras pelas quais os alunos respondem a essas configurações acadêmicas alternativas.

O trabalho de Rosário, Morão e Chaleta (2005) analisou as relações entre o perfil de comportamentodo estudante e os Trabalhos a serem Resolvidos em Casa (TPC). Constatou que ao nível instrutivo dos pais associa-se, positiva e significativamente, os perfis de atitudes e comportamentos de TPC. As atitudes e comportamentos de TPC correlacionam-se, positiva e significativamente, com os perfis autorregulatório, diante do estudo. Alunos que se percepcionam como mais autoeficazes registam melhores perfis de atitudes e comportamentos em TPC. 


\subsection{Modelo sociocognitivo de Bandura}

O modelo sociocognitivo de explicação do comportamento do indivíduo proposto por Bandura foi uma reação contra os modelos da psicanálise e dos modelos behavioristas. Nesse sentido, o referido modelo enfatizou as interações de fatores comportamentais, pessoais e ambientais que influenciam o comportamento e na motivação por meio do modelo da Casualidade Triárquico Recíproco na interação entre comportamento, pessoa e ambiente (Bandura, 1986).

Com base na teoria Sociocognitiva, o desenvolvimento humano ocorre por meio de adaptações e mudanças no indivíduo, de modo que este se torne proativo, autorreflexivo, autoorganizado e autorregulado (Bandura, 2008). Nessa perspectiva, Bandura (2001, 2006, 2008) apontou quatro características do comportamento do indivíduo: a intenção na realização de uma ação futura, a elaboração de planos e de estratégias; a antecipação de futuras ações, ao traçar objetivos e prevendo os possíveis resultados (guia comportamental); a autorreação ao autorregular seus comportamentos por meio de metas e padrões pessoais que refletem a finalização, ou não, de uma tarefa (relação com a percepção de autoeficácia); e a autorreflexão, em que há um julgamento sobre si (pensamentos e ações).

Para Bandura (1986, 2001, 2006, 2008), o desenvolvimento pessoal sofre influências sociais e estruturais ao mesmo tempo em que influencia o seu contexto social. No desenvolvimento do modelo social, Bandura (1986) indica que as fases relacionam as influências no comportamento do observador; a de prover respostas rápidas a questões sociais; prover o aprendizado pela observação para aquisição de competências cognitivas e novos hábitos comportamentais.

Nesse contexto, o modelo Triárquico, de Bandura, relaciona com o constructo da aprendizagem autorregulada devido às interações pessoais (acontecimentos cognitivos, afetivos e biológicos), ambientais (variedade de influências sociais do cotidiano), contextuais e comportamentais (padrões de atividade) em que se adquire novas aprendizagens. Concebe-se que nesse modelo existam influências reciprocas entre os vários fatores, porém sem padrões de interação, dado que cada fator pode ser variável ao contexto em que esteja inserido.

Segundo Castro (2007), com base na teoria Sociocognitiva, a autorregulação da aprendizagem vai além da compreensão dos fatores pessoais, pois considera o ambiente físico e social do aprendente, pois considera que é no ambiente social que ocorre a instrução e a modelagem, que funcionam de condução para transmitir competências autorregulatórias, como a persistência, autoelogios e autorreações, através dos pais, professores, pares e comunidade (Zimmerman, 2000a).

\subsection{Teoria da Autorregulação do Aprendizado, de Zimmerman}

A teoria da Autorregulação elaborada por Zimmerman é influenciada pela teoria Sociocognitiva, de Bandura. Para Zimmerman (2002), a autorregulação do aprendizado é um processo autodiretivo em que os aprendizes utilizam de habilidades mentais em prol de competências acadêmicas. Para Zimmerman $(2000 ; 2002)$, processo de autorregulação acontece de modo gradual e contínuo, em que a dependência de apoio social é gradativamente reduzida e, para isso, mobiliza a observação, emulação, autocontrole e a autorregulação.

O modelo de Autorregulação da Aprendizagem proposta por Zimmerman acontece em três fases de forma cíclica: a primeira fase é a cíclica, considerada a fase prévia do desempenho (planejamento); a segunda fase é a do desempenho ou controle volitivo (realização); e, por fim, a fase a autorreflexão (avaliação); em que cada uma dessas fases mobilizam cognições distintas no estudante e funciona de modo dinâmico, cíclico e aberto (Rosário, 2002; Schunk \& Zimmerman, 2003; Silva, 2004; Zimmerman, 1998, 2002; Zimmerman \& Schunk, 2004; Schunk, Pintrich \& Meece, 2008). 
Nesse contexto, Zimmerman e Martinez-Ponz (1986) identificaram quatorze possíveis estratégias mais utilizadas por alunos autorregulados, que, segundo os autores, o uso destas estratégias confere ao aluno um valioso ferramental de aprendizado e sua utilização é diretamente relacionada com índices de sucesso acadêmico. As estratégias presentes em alunos autorregulados, com suas respectivas definições e exemplos estão apresentadas no Quadro 1.

\begin{tabular}{|c|c|c|c|}
\hline & Estratégias & Definição & Exemplos: (Rosário, 1999) \\
\hline 1 & Autoavaliação & $\begin{array}{l}\text { Declarações que indicam as avaliações dos alunos } \\
\text { sobre a qualidade ou progresso do seu trabalho. }\end{array}$ & $\begin{array}{l}\text { "...verifiquei o meu trabalho para ter } \\
\text { a certeza que estava bem". }\end{array}$ \\
\hline 2 & $\begin{array}{l}\text { Organização e } \\
\text { transformação }\end{array}$ & $\begin{array}{l}\text { Declarações que indicam as iniciativas dos alunos } \\
\text { para reorganizarem, melhorando-os, os materiais } \\
\text { de aprendizagem. }\end{array}$ & $\begin{array}{l}\text { "...faço sempre um esquema } \\
\text { antes de realizar os relatórios das } \\
\text { experiências de química". }\end{array}$ \\
\hline 3 & $\begin{array}{l}\text { Estabelecimento } \\
\text { de objetivos e } \\
\text { planejamento }\end{array}$ & $\begin{array}{l}\text { Declarações indicando o estabelecimento de objetivos } \\
\text { educativos: planejamento, fase no tempo e conclusão } \\
\text { de atividades relacionadas com esses objetivos. }\end{array}$ & $\begin{array}{l}\text { "... começo a estudar duas semanas } \\
\text { antes do teste e fico descansada". }\end{array}$ \\
\hline 4 & $\begin{array}{l}\text { Procura de } \\
\text { informação }\end{array}$ & $\begin{array}{l}\text { Declarações indicado os esforços dos alunos para } \\
\text { adquirir informações extra de fontes não sociais } \\
\text { quando enfrentaram uma tarefa escolar. }\end{array}$ & $\begin{array}{l}\text { “...antes de começar um trabalho, } \\
\text { vou a biblioteca da escola recolher } \\
\text { o máximo de informações sobre o } \\
\text { tema". }\end{array}$ \\
\hline 5 & $\begin{array}{l}\text { Tomada de } \\
\text { apontamentos }\end{array}$ & $\begin{array}{l}\text { Declaração indicando os esforços para registrar } \\
\text { eventos ou resultados }\end{array}$ & $\begin{array}{l}\text { “...nas aulas absorvo o máximo } \\
\text { de apontamentos sobre o que o } \\
\text { professor dá". }\end{array}$ \\
\hline 6 & $\begin{array}{l}\text { Estrutura } \\
\text { ambiental }\end{array}$ & $\begin{array}{l}\text { Declarações indicando esforços para selecionar ou } \\
\text { alterar o ambiente físico ou psicológico de modo a } \\
\text { promover a aprendizagem. }\end{array}$ & $\begin{array}{l}\text { “...para não me distrair, isolo-me no } \\
\text { quarto" ou “...para me concentrar no } \\
\text { que estou fazendo, desligo o som". }\end{array}$ \\
\hline 7 & Autoconsequência & $\begin{array}{l}\text { Declarações indicando a imaginação ou a } \\
\text { concretização de recompensas ou punições para } \\
\text { sucessos ou fracassos escolares. }\end{array}$ & $\begin{array}{l}\text { "...se me der bem no teste, compro } \\
\text { uns chocolates". }\end{array}$ \\
\hline 8 & $\begin{array}{l}\text { Repetição e } \\
\text { memorização }\end{array}$ & $\begin{array}{l}\text { Declarações indicando as iniciativas e os esforços } \\
\text { dos alunos para memorizar o material. }\end{array}$ & $\begin{array}{l}\text { “...na preparação de um teste } \\
\text { de física, escrevo muitas vezes a } \\
\text { formula, até saber de cor" }\end{array}$ \\
\hline $9-11$ & $\begin{array}{l}\text { Procura de ajuda } \\
\text { social }\end{array}$ & $\begin{array}{l}\text { Declarações indicando as iniciativas e os esforços } \\
\text { dos alunos para procurarem ajuda dos pares (9); } \\
\text { professores (10); e adultos (11) }\end{array}$ & $\begin{array}{l}\text { "... se tenho dificuldades no estudo } \\
\text { peço ajuda ao meu pai que é médico" }\end{array}$ \\
\hline $\begin{array}{l}12- \\
14\end{array}$ & Revisão de dados & $\begin{array}{l}\text { Declarações indicando os esforços-iniciativas dos } \\
\text { alunos para relerem as notas (12); teste (13); e livros } \\
\text { de texto (14) a fim de se prepararem para uma aula } \\
\text { ou exercício escritos. }\end{array}$ & $\begin{array}{l}\text { “...antes dos testes revejo sempre os } \\
\text { resumos da matéria que fiz" ou "Para } \\
\text { me preparar para um teste resolvo os } \\
\text { enunciados dos que já fiz". }\end{array}$ \\
\hline
\end{tabular}

Fonte: Zimmerman e Martinez-Pons (1986, p. 5-16); Rosário (2001, p.52), adaptado.

Quadro 1. Estratégias de aprendizado autorregulado

No estudo de Silva e Simão (2016), foi analisada a possibilidade da eficácia da entrevista como tarefa pode auxiliar e potencializar a identificar processos autorregulatórios na aprendizagem, como potenciá-los nos aprendizes. Os autores concluíram que ocorreu a evolução nos perfis de aprendizagem, já que os alunos passaram a adequar os objetivos das estratégias, interiorizar normas de ação e fazer revisão. Este estudo permite conferir a importância para a estratégia E10, de "revisão dos dados", que, para Zimmerman e Martinez-Pons (1986), é por meio dela que ocorrem os esforços e iniciativas dos alunos para relerem as notas, testes, livros de texto, apontamentos a fim de se prepararem para alguma aula ou exercícios escritos.

Já Frison (2016), constatou que a monitoria propiciou o uso de diferentes estratégias para potencializar a aprendizagem, e que o pequeno grupo de estudo proporcionou uma verdadeira aprendizagem, pois um ajudaram aos outros, o que propiciou maior empenho e dedicação nas tarefas. Percebe-se, claramente, no estudo realizado a presença da estratégia de aprendizagem de "ajuda social" (E9), em que há o suporte a esclarecimento de dúvidas dos discentes por meio daqueles estudantes mais avançados no curso. 


\section{Metodologia}

A pesquisa é exploratória visto que pretende maior compreensão sobre os fatores que podem afetar a aprendizagem autorregulada; e descritivo, pois pretendeu descrever os fenômenos da aprendizagem autorregulada. Para Gil (2007), as pesquisas exploratórias têm como objetivo proporcionar maior familiaridade com o problema, com vistas a torná-lo mais explícito ou a construir hipóteses. No que se refere aos procedimentos, pode ser enquadrado como bibliográfico devido à varredura da literatura necessária em qualquer construção científica; de campo, pois houve a aplicação de questionários aos discentes de Contabilidade com intuito de colher informações para o desenvolvimento do estudo; e survey, pois buscaram-se informações sobre as características demográficas, acadêmicas e sobre o uso das estratégias de autorregulação. A abordagem do problema foi classificada como quantitativa, pois foram utilizados diversos métodos estatísticos com propósito de responder aos objetivos do estudo, como a análise múltipla e análise fatorial.

A seleção da amostra envolveu critérios que permitisse atender aos objetivos da pesquisa. Nesse sentido, foram escolhidas duas instituições públicas e uma instituição privada sem fins lucrativos, tradicionais na oferta do curso de Contabilidade no município de Salvador -Bahia e que facilitaram o acesso à IES.

A proposta do modelo está ancorada na ideia que variáveis demográficas e acadêmicos podem influenciar a Aprendizagem Autorregulada dos discentes. As variáveis demográficas são representadas pelo gênero, idade, semestre, tempo de estudo, se o estudante estagia ou trabalha (em horas), escolaridade do pai e da mãe; e as acadêmicas pelo semestre em curso e o tipo de IES (se pública ou privada).

Ressalta-se que os dados foram colhidos de duas formas: por meio da aplicação presencial na Fundação Visconde de Cairu (FVC) e Universidade do Estado da Bahia (UNEB); e, na Universidade Federal da Bahia, os dados foram colhidos com auxílio da plataforma on-line do Survey Monkey. O envio do questionário on-line foi encaminhado pela coordenação do curso de Ciências Contábeis, da UFBA, já que apenas o corpo administrativo da instituição possui o acesso a todos os e-mails dos alunos. A amostra correspondeu a 713 discentes de Contabilidade correspondentes de uma população de 1.929 alunos matriculados no segundo semestre de 2017.

O instrumento de coleta de dados foi o questionário segmentado em dois blocos. Nesse sentido, foram utilizados oito quesitos demográficos e acadêmicos, dez questões que abordaram sobre as estratégias de aprendizado autorregulado identificados por Zimmerman (1986). No bloco I constam as questões relacionadas às características socioeconômicas e acadêmicas do estudante: (a) gênero, (b) idade, (c) nome da faculdade em que estuda, (d) semestre em curso, (e) tempo de estudo diário, (f) escolaridade do pai, (g) escolaridade da mãe e (h) carga horária de estágio/trabalho. Para identificá-las foram utilizados oito quesitos contemplados em estudos anteriores (Zimmerman \& Martinez-Pons, 1986; Rosário ,1999; Filcher \& Miller, 2000; Zimmermann, 2002; Rosário \& Almeida, 2005; Schunk, 2005; Bell \& Akroyd, 2006).

O bloco II buscou verificar o uso de estratégias de aprendizagem autorreguladas identificadas por Zimmerman e Martinez-Pons (1986). Nesse sentido, o bloco é composto por 10 quesitos que apresentam as variáveis de Autoavaliação; Organização e transformação; Estabelecimento de objetivos e planejamento; Procura de informação; Tomada de apontamentos; Estrutura de ambiental; Autoconsequência; Repetição e memorização; Procura de ajuda social e Revisão de dados. O bloco II do questionário correspondeu às características identificadas por Zimmerman (1986) e foi utilizado escala do Likert com sete pontos, cujos extremos são: 1 (nunca realiza a atividade) e 7 (sempre realiza a atividade). O uso da escala do Likert tem o propósito de evidenciar a relevância atribuída pelos estudantes às questões propostas e as variáveis a ela relacionadas (Silva Júnior \& Costa, 2014, p. 5).

Foram testadas duas hipóteses neste estudo com objetivo de contribuir para esclarecer as relações entre a aprendizagem autorregulada e fatores sociais e acadêmicos no curso de Ciências Contábeis: H1: Variáveis sociais podem explicar a autorregulação da aprendizagem e H2: Variáveis acadêmicas podem explicar a autorregulação da aprendizagem. 
Com o objetivo de investigar como a aprendizagem autorregulada dos estudantes podem se relacionar com fatores demográficos e acadêmicos (objetivo c) e testar as hipóteses $\mathrm{H}_{1}$ e $\mathrm{H}_{2}$ desse estudo, foi utilizada a regressão múltipla. De acordo com Hoffmannt (2006), tem-se uma regressão múltipla quando se admite que a variável dependente é uma função linear de duas ou mais variáveis explanatórias e que pode ser expressa como mostra a equação: $\mathbf{Y}=\boldsymbol{\beta} \mathbf{0}+\boldsymbol{\beta} \mathbf{1} \mathrm{X}_{1}+\boldsymbol{\beta} \mathbf{2} \mathrm{X}_{2}+\boldsymbol{\beta} 3 \mathrm{X}_{3}+\ldots . \boldsymbol{\beta} \mathbf{n} \mathbf{X}_{\mathrm{n}}+\boldsymbol{\varepsilon}$

Os modelos que permitiram averiguar essas relações estão descritas no Quadro 2.

\begin{tabular}{ll}
\hline Modelo SRL Demográfico & $\mathrm{SRLi}=\beta_{0}+\beta 1$ Gen $+\beta 2$ Epai $+\beta 3$ Emae $+\beta 4$ Tre $+\beta 5 / d a+\beta 6$ Tes $+\varepsilon$ \\
\hline Modelo SRL Acadêmico & $\mathrm{SRLi}=\beta_{0}+\beta 1 \mathrm{Sem}+\beta 2$ IES $+\varepsilon$ \\
\hline Modelo SRL demográficos e acadêmicos & $\mathrm{SRLi}=\beta_{0}+\beta 1$ Gen $+\beta 2$ Epai $+\beta 3$ Emae $+\beta 4$ Tre $+\beta 5 / d a+\beta 1$ Sem $+\beta 2$ IES $\beta 6$ TeS $+\varepsilon$ \\
\hline
\end{tabular}

Legenda: SRLi = índice de Aprendizagem Autorregulada (média das respostas da escala, 1 a 7, de aprendizado autorregulado) $\beta_{0}=$ Constante $\beta 1$ Gen = Gênero $\beta 2$ Epai $=$ Escolaridade do Pai $\beta 3$ Emae $=$ Escolaridade da Mãe $\beta 4$ Tre $=$ Trabalho ou estágio $\beta 5 / d a=$ Idade $\beta 6$ Tes $=$ Tempo de estudo $\beta 1 \mathrm{Sem}=$ Semestre $\beta 2$ IES = Tipo de IES $\varepsilon=$ Erro ou resíduo da regressão.

Fonte: elaborado pelo autor (2018).

Quadro 2. Modelos de regressão multipla

O modelo SRL demográficos e acadêmicos teve por objetivo analisar as relações "relação simultânea entre a aprendizagem autorregulada e fatores demográficos e acadêmicos", e foi construído mais um cenário. A ideia subjacente é que os dois fatores podem explicar, simultaneamente, a aprendizagem autorregulada do aluno.

\section{Resultados}

Esse estudo teve como objetivo geral verificar que fatores demográficos e acadêmicos influenciam a aprendizagem autorregulada nas IES de Salvador (BA) pesquisadas. Para tanto, foram coletados dados de uma amostra de 713 discentes de Instituição de Ensino Superior localizadas em Salvador, Bahia. A Tabela 1 evidencia que a amostra foi composta por $67,6 \%$ de estudantes instituições de ensino público e 32,4\% de IES privada. Da composição de IES Pública, 37,2\% dos estudantes são da Universidade Federal da Bahia (UFBA), 30,4\% da Universidade do Estado da Bahia (Uneb).

Tabela 1

Composição da amostra por IES

\begin{tabular}{ccccc}
\cline { 2 - 5 } & Tipo de IES & IES & Fi & Fi\% \\
\cline { 2 - 5 } & \multirow{2}{*}{ Pública } & UFBA & 265 & 37,2 \\
\cline { 3 - 5 } & & UNEB & 217 & 30,4 \\
\hline Sub-total & & & 482 & 67,6 \\
\hline \multirow{2}{*}{ Total } & Privada & FVC & 231 & 32,4 \\
\hline
\end{tabular}

Fonte: Dados da pesquisa, 2018.

Com relação ao gênero desses alunos, 44,74\% são do sexo masculino e 55,26\% do gênero feminino. Desses, $16,41 \%$ possuem idade até vinte anos, $42,36 \%$ entre 21 a $25,17,95 \%$ entre 26 a 30, 13,88\% entre 31 a 35 e 9,4\% possuem idade superior a 36 anos. Quanto aos semestres matriculados no curso de Ciências Contábeis, $44,6 \%$ estão cursando até o quarto semestre e 55,4\%, do quinto ao oitavo semestre. 
Para atingir o primeito objetivo específico desse estudo, foram utilizadas as estratégias de aprendizagem autorreguladas identificadas por Zimmerman e Martinex-Pons (1986), apresentadas e exemplificada no Quadro 1. Por meio da Tabela 2, que adota um ponto de segmentação em até três e maior que três, verifica-se que as estratégias mais utilizada pelos estudantes de Contabilidade foram de autoavaliação (E1), a procura de ajuda social (E9), estratégia de estrutura ambiental (E6) e estratégia de revisão de dados (E10). Esses achados são convergentes achados de Zimmerman $(1986 ; 1989)$ ao indicar que os alunos tendem a usar preferencialmente a estratégia da autoavaliação, enquanto as estratégias menos utilizadas foram a estratégia autoconsequência (E7) e estratégia de estabelecimento de objetivos e planejamento (E3).

Tabela 2

Uso de estratégias de autorregulação da aprendizagem por estudantes de contabilidade, por meio de um ponto de segmento

\begin{tabular}{llcccccccccc} 
& & E1 & E2 & E3 & E4 & E5 & E6 & E7 & E8 & E9 & E10 \\
\hline$<4$ & $\mathrm{Fi}$ & 52 & 136 & 413 & 158 & 114 & 82 & 524 & 223 & 65 & 91 \\
& $\mathrm{Fi} \%$ & 7,29 & 19,07 & $\mathbf{5 7 , 9 2}$ & 22,16 & 15,99 & 11,50 & $\mathbf{7 3 , 4 9}$ & 31,28 & 9,12 & 12,76 \\
\hline $\mathbf{2} 4$ & $\mathrm{Fi}$ & 661 & 577 & 300 & 555 & 599 & 631 & 189 & 490 & 648 & 622 \\
& $\mathrm{Fi} \%$ & $\mathbf{9 2 , 7 1}$ & 80,93 & 42,08 & $\mathbf{7 7 , 8 4}$ & 84,01 & $\mathbf{8 8 , 5 0}$ & 26,51 & 68,72 & $\mathbf{9 0 , 8 8}$ & $\mathbf{8 7 , 2 4}$ \\
\hline
\end{tabular}

Fonte: dados da pesquisa (2018).

Com exceção das estratégias autoconsequência (E7) e estabelecimento de objetivos e planejamento (E3), percebe-se que todas as outras estratégias de autorregulação foram apontadas com uso superior a 68\%. Silva et al. (2017) encontraram resultados semelhantes para discentes de IES privada no Ensino a Distância privada de Contabilidade e Lima Filho et al. (2015) em duas IES públicas do Estado da Bahia para o uso das estratégias E1, E6 e E10.

A Tabela 2 evidencia o forte uso da estratégia da autoavaliação (92,71\%), apontada como a mais utilizada pelos estudantes de Contabilidade. O uso da estratégia da autoavaliação permite que o estudante corrija eventuais erros cometidos em tarefas e aperfeiçoe na próxima tarefa. Sobre o uso da autoavaliação no processo educativo, Ribeiro (2003) enfatiza que se trata de uma importante estratégia de prática dos estudantes, pois permite avaliar o que aprendeu.

A segunda estratégia mais usual foi a de "procura de ajuda social" (E9), com 90,88\%. Compreende-se que se trata de uma estratégia fundamental para se obter sucesso na realização de uma tarefa e que o uso frequente dos estudantes de Contabilidade repercute em melhores rendimentos acadêmicos. Na pesquisa realizada por Frison (2016), à luz da aprendizagem autorregulada, foi constatado que a monitoria propiciou o uso de diferentes estratégias para potencializar a aprendizagem.

A estratégia de "estrutura ambiental" (E6) foi apontada por 88,50\%. Para Corno (2001), o aprendizado autorregulado pode ser aumentado pelas alterações efetuadas a uma determinada tarefa que pode ser realizada pelo próprio indivíduo ou reações do ambiente circundante, pois considera-se que o indivíduo é influenciado pelas expectativas e intenções que impactam a motivação e emoção. Rosário e Polydoro (2012), acreditam que os estudantes são capazes de fazer uso de uma série de estratégias volitivas orientadas para evitar distrações externas e internas, para manter a concentração, o esforço e a motivação durante a realização das tarefas. Portanto, entende-se que os estudantes analisados utilizam de estruturas essenciais para promover uma boa aprendizagem e assim serem mais autorregulados.

A não utilização da estratégia E7, "autoconsequência" (26,51\%), tem sido recorrente nos estudos sobre autorregulação da aprendizagem em alunos de Ciências Contábeis, tanto para a modalidade "presencial" quanto "a distância” (Silva et al., 2017; Lima Filho, Lima \& Bruni, 2015; Aguiar \& Silva, 2015; 2017). A literatura sobre autorregulação da aprendizagem apontada por Zimmerman e Martinez-Pons (1986) indica que as quatorze estratégias integram o processo cíclico maior segmentadas nas três fases (planejamento, execução e autorreflexão), onde estas são fases complementares e continuas. Acredita-se que os estudantes 
possam estar utilizando de outras formas de incentivos para cumprir a tarefa, que configure mais a realidade cultural brasileira. Com base nos estudos anteriores e nos resultados desse estudo, defende-se que a autoconsequência não seja uma estratégia de autorregulação da aprendizagem presente em estudantes de Contabilidade em IES no contexto brasileiro (Silva et al., 2017, Aguiar \& Silva, 2017, Krauss, Silva \& Zonatto, 2017, Lima Filho et al. 2015), entre as identificadas por Zimmerman e Martinez-Pons (1986).

A estratégia E3, de "estabelecimento de objetivos" e planejamento (42,08\%) é a segunda menos utilizada pelos discentes. Acredita-se que o pouco uso dessa estratégia possa estar relacionada ao acúmulo de atividades semanais, incluindo os diversos trabalhos, provas, seminários aliados ao pouco tempo disponível para dedicar as atividades acadêmicas. Por outro lado, acredita-se que seja altamente prejudicial para o cumprimento da tarefa estabelecida, uma vez que não ocorre a preparação e estabelecimento de estratégias para conseguir sucesso. Esse resultado converge para os achados de Polydoro e Rosário (2012) que identificaram pouca utiliza na estratégia E3 e constataram que a procrastinação acadêmica na preparação dos estudos se relaciona de forma positiva com a autorregulação, ou seja, quanto mais discentes adiam os estudos para realização de uma atividade ou avaliação menor será sua percepção de autorregulação na aprendizagem.

O segundo objetivo especifico identificou as dimensões associadas às estratégias de aprendizado autorregulado adotadas pelos estudantes de Ciências Contábeis. Hair, Black, Babin, Anderson e Tatham (2006) sugerem que a amostra deve ser superior a 50 observações, sendo aconselhável no mínimo 100 casos para assegurar resultados mais robustos. A razão entre o número de casos e a quantidade de variáveis deve exceder cinco para um ou mais (Hair et al., 2006). Esse estudo analisou 713 casos e foram consideradas 10 variáveis, cumprindo ambos pré-requisitos para a aplicação do teste. Na Tabela 3 é possível verificar os componentes principais da análise fatorial para as estratégias de aprendizagem autorreguladas.

Tabela 3

Análise dos componentes principais para as estratégias de aprendizagem autorregulada

\begin{tabular}{|c|c|c|c|c|c|c|c|c|c|}
\hline & Total & $\begin{array}{c}\text { \% de } \\
\text { variância }\end{array}$ & $\begin{array}{c}\% \\
\text { Acumulativa }\end{array}$ & Total & $\begin{array}{c}\text { \% de } \\
\text { variância }\end{array}$ & $\begin{array}{c}\% \\
\text { Acumulativa }\end{array}$ & Total & $\begin{array}{c}\text { \% de } \\
\text { variância }\end{array}$ & $\begin{array}{c}\% \\
\text { Acumulativa }\end{array}$ \\
\hline 1 & 2,935 & 29,345 & 29,345 & 2,935 & 29,345 & 29,345 & 2,212 & 22,118 & 22,118 \\
\hline 2 & 1,164 & 11,639 & 40,984 & 1,164 & 11,639 & 40,984 & 1,887 & 18,866 & 40,984 \\
\hline 3 & ,967 & 9,674 & 50,659 & & & & & & \\
\hline 4 & ,896 & 8,957 & 59,615 & & & & & & \\
\hline 5 & ,833 & 8,334 & 67,949 & & & & & & \\
\hline 6 & ,762 & 7,621 & 75,570 & & & & & & \\
\hline 7 & ,731 & 7,313 & 82,883 & & & & & & \\
\hline 8 &, 630 & 6,298 & 89,181 & & & & & & \\
\hline 9 &, 557 & 5,566 & 94,747 & & & & & & \\
\hline 10 & ,525 & 5,253 & 100,000 & & & & & & \\
\hline
\end{tabular}

Fonte: dados da pesquisa, 2018

A análise da Tabela 3 aponta a presença de dois fatores para as estratégias de aprendizagem autorregulada que explicam 40,98\% da variabilidade total. Para Hair et al. (2006) e Shimada, Chiusoli e Messetti (2010) um critério na retenção de fatores é o teste de Kaiser, que considera os valores superiores a 1,0 (eigenvalue $>1$ ). Na observância dos dados, o primeiro fator (eigenvalue $=2,93$ ) consegue explicar 29,34\% da variância total e o segundo (eigenvalue $=1,16$ ) com 11,64\%.

De acordo com Catell (1996) e Menezes (2006), que a determinação do fator finalize é necessário verificar as afinidades entre os itens. Esses últimos autores asseguram a necessidade de expurgar os fatores que apresentem carga fatorial menor que 0,32 , aqueles que apresentem cargas fatoriais similares em dois ou mais fatores com diferenças inferior a $0,10 \mathrm{e} o$ fator que apresente apenas um item. Os resultados da rotação oblíqua estão apresentados na Tabela 4. 
Tabela 4

Análise dos coeficientes de correlação por meio do método Pattern Matrix

\begin{tabular}{ccccccccccc}
\hline Dimensões & E1 & E2 & E3 & E4 & E5 & E6 & E7 & E8 & E9 & E10 \\
\hline 1 &, 328 &, 440 &,- 055 &, 079 &, 377 & $\mathbf{, 4 2 4}$ &,- 629 & $\mathbf{, 7 1 6}$ & $\mathbf{5 0 0}$ & $\mathbf{6 5 2}$ \\
\hline 2 & $\mathbf{6 1 1}$ &, 535 & $\mathbf{6 9 1}$ & $\mathbf{, 7 0 2}$ &,- 428 &, 219 &, 362 &,- 001 &, 355 &, 306 \\
\hline
\end{tabular}

Fonte: dados da pesquisa, 2018

Foi utilizado o método da rotação oblíqua Promax no intuito de sondar os coeficientes de correlação. Por meio da rotação Promax, é fornecida uma matriz padrão e outra de estrutura (inclusive a matriz fatorial), que apresenta clusters de intercorrelações entre as variáveis oblíquas que são agrupadas de acordo a carga fatorial, ou seja, segundo o grau de envolvimento com os padrões (Catell, 1966). Por meio da Pattern Matrix, é possível indicar aqueles fatores que estão fortemente envolvidos quando se observam as cargas fatoriais em cada agrupamento (cluster) (Shimada et al., 2010).

Segundo Menezes (2006), a conclusão da análise fatorial chega ao fim quando são examinadas as afinidades dos itens com os fatores do modelo. As variáveis de um modelo fatorial são expurgadas segundo os seguintes critérios: (a) quando o valor absoluto da carga fatorial principal do item é menor do que 0,32; (b) quando existem cargas fatoriais similares em dois ou mais fatores em um mesmo item (a diferença entre valores absolutos das cargas fatoriais dos itens é menor do que 0,10); e (c) um fator é formado por dois ou mais itens (Menezes, 2006).

Com esse contexto, o item estratégia "organização e transformação" (E2) foi excluído da análise por apresentar carga fatorial semelhantes na primeira, e na segunda dimensão menor que 0,10 . As estratégias (E5) e (E7) também foram excluídas devido o valor absoluto da carga principal do item é menor que 0,32 , critério (a). Após a referida análise e exclusão, permaneceu a existência de dois fatores que agregam as referidas estratégias de autorregulação de aprendizagem: o Fator um, que agregou os itens "estratégia de estrutura ambiental" (E6), "estratégia de repetição e memorização" (E8), "estrutura de procura por ajuda" (E9) e "estratégia de revisão de dados" (E10). E o segundo fator que foi composto pela "estratégia de autoavaliação" (E1), "estratégia de estabelecimento de objetivos e planejamento" (E3) e "estratégia de procura de informação" (E4). As dimensões encontradas foram intituladas como: Fator 1 "Dimensão de Execução" e o Fator 2 "Dimensão de planejamento e reação".

Após encontrar as dimensionalidades e convergências relacionadas as repostas da amostra sobre as estratégias de autorregulação da aprendizagem, as dimensões foram validadas segundo os critérios Netemeyer et al (2003), por meio do índice Kaiser-Meyer-Olkin (KMO), do teste de Esfericidade, de Bartlett, e do Alfa, de Cronbach. Os resultados apontaram KMO aceitável (0,698 e Qui-quadrado =292,276); esfericidade, de Bartlett, significativo (0,000); Alfa, de Cronbach, confiável (0,671); e coeficientes, de Pearson, positivos e significativos.

Na Tabela 5, é possível verificar as médias das dimensões de utilização das estratégias de aprendizado autorregulado, alcançado por meio da estatística descritiva. Percebe-se que, as dimensões de organização e preparação são mais utilizadas pelos estudantes que a de fixação e autorreação e que, em geral, as estratégias autorreguladas são utilizadas moderadamente pelos estudantes de Contabilidade. $\mathrm{O}$ baixo desvio-padrão $(0,041101$ e 0,03862$)$ assegura que se trata de uma amostra homogênea.

Tabela 5

Estatística descritiva para as dimensões do aprendizado autorregulado

\begin{tabular}{lccccc}
\multicolumn{1}{c}{ Dimensões } & N & Mínimo & Máximo & Média & Desvio padrão \\
\hline Execução & 713 & 1,00 & 7,00 & 5,2847 & 0,04110 \\
\hline Planejamento e reação & 713 & 1,00 & 6,33 & 4,6639 & 0,03862 \\
\hline Fonte: dados da pesquisa (2018). & & & & &
\end{tabular}

Fonte: dados da pesquisa (2018). 
Essa seção buscou verificar as dimensões associadas à utilização de estratégias de autorregulação por meio da Análise Fatorial. Foram encontradas duas dimensões que denominadas de "execução", de "planejamento e reação".

O último objetivo desse estudo analisou a relação entre aprendizagem autorregulada e as variáveis demográficas e acadêmicas de estudantes de Contabilidade das Instituições de Ensino Superior de Salvador (BA). Antes de realizar a regressão múltipla e verificar as relações entre as variáveis e para chegar a resultados robustos e consistentes, faz-se necessário realizar alguns testes preliminares, a fim de sondar se as variáveis apresentam os pressupostos básicos para se realizar uma regressão múltipla. Os pressupostos testados foram: Normalidade - verifica se a variável dependente ou resposta apresenta uma distribuição normal; Autocorrelação - analisa a correlação entre as variáveis; Multicolinearidade - verifica possíveis problemas de multicolinearidade.

Análise do primeiro pressuposto apresentou distribuição normal em "formato de sino" para a variável resposta ou dependente, satisfazendo ao primeiro pressuposto. $\mathrm{O}$ teste Kolmogoroy-Smirnov apontou sig. de 0,88 e assim, como foi maior que 0,05 , aceita-se a hipótese de normalidade dos dados. O segundo pressuposto analisou a correlação entre as variáveis desse estudo por meio do coeficiente de Correlação, de Pearson. Dancey e Reidy (2006) para a seguinte classificação para a análise de Pearson: $r=0,10$ até 0,30 (fraco); $\mathrm{r}=0,40$ até 0,6 (moderado); $\mathrm{r}=0,70$ até 1 (forte). Os dados revelaram valores moderados. Em geral, os valores da correlação foram significantes e não apresentaram altos valores. Foi também verificado o teste de VIF, que, segundo Bruni (2012, p.240), indica se "os coeficientes e testes podem conduzir a conclusões erradas, caso as variáveis exógenas, independentes, apresentem altas correlações cruzadas" e que, segundo Miloca (2002), o valor de VIF não pode ser superior a quatro, embora alguns autores tolerem cinco. Os resultados satisfizeram o indicado pela literatura, assim, não apresentando problemas de multicolinearidade, o que permitiu analisar os resultados das regressões.

A Tabela 6 apresenta os resultados dos cenários quando analisada a influência dos fatores demográficos ao considerar como variável dependente os fatores gerados pela análise fatorial (fator execução; e planejamento e reação) e o índice médio da Aprendizagem autorregulada.

Tabela 6

Análise múltipla dos Fatores Demográficos

\begin{tabular}{|c|c|c|c|c|c|c|c|c|c|}
\hline \multicolumn{4}{|c|}{ Fator "execução" } & \multicolumn{3}{|c|}{ Fator "planejamento e reação" } & \multicolumn{3}{|c|}{ Índice SRL } \\
\hline Variáveis & Coeficiente & Estatística-t & sig. & Coeficiente & Estatística-t & sig. & Coeficiente & Estatística-t & sig. \\
\hline (Constante) & 4,772 & 18,803 & 0 & 4,19 & 17,526 & 0 & $-0,282$ & $-1,744$ & 0,082 \\
\hline Gênero & 0,154 & 1,887 & 0,06 & 0,3 & 3,915 & 0 & 0,143 & 2,755 & 0,006 \\
\hline Idade & $-0,067$ & $-1,819$ & 0,069 & $-0,057$ & $-1,65$ & 0,099 & $-0,057$ & $-2,448$ & 0,015 \\
\hline $\begin{array}{l}\text { Tempo de } \\
\text { estudo }\end{array}$ & 0,226 & 6,041 & 0 & 0,169 & 4,807 & 0 & 0,156 & 6,545 & 0 \\
\hline $\begin{array}{l}\text { Escolaridade } \\
\text { do Pai }\end{array}$ & $-0,063$ & $-2,402$ & 0,017 & $-0,032$ & $-1,29$ & 0,197 & $-0,049$ & $-2,952$ & 0,003 \\
\hline $\begin{array}{l}\text { Escolaridade } \\
\text { da Mãe }\end{array}$ & 0,046 & 1,719 & 0,086 & $-0,009$ & $-0,338$ & 0,735 & 0,016 & 0,916 & 0,36 \\
\hline $\begin{array}{l}\text { Trabalho ou } \\
\text { estágio }\end{array}$ & $-0,029$ & $-1,038$ & 0,3 & $-0,035$ & $-1,339$ & 0,181 & $-0,019$ & $-1,107$ & 0,269 \\
\hline $\mathrm{N}$ & 713 & & & 713 & & & 713 & & \\
\hline $\mathrm{R}^{2}$ & 0,08 & & & 0,079 & & & 0,105 & & \\
\hline F (sig.) & 10,246 & & & 10,142 & & & 13,788 & & \\
\hline
\end{tabular}

Fonte: Dados da pesquisa, 2018. 
Os resultados permitem indicar que as variáveis demográficas que influenciam positivamente o aprendizado autorregulado foi o tempo de estudo; e a escolaridade do pai influencia negativamente. As variáveis escolaridade da mãe, gênero, trabalho e idade não tiveram significância no modelo. Esse achado converge com os estudos de Freitas (2005), que apontou que aqueles estudantes que estudam na véspera de provas tendem a ser menos autorregulado que aqueles que estudam diariamente. Os teóricos da autorregulação indicam que a aprendizagem é uma experiência pessoal que requer participação ativa, dedicada e informada por parte dos alunos (Zimmerman, Bonner \& Kovach, 1996) - e que o planejamento do tempo diário promove a fixação das competências para alcançar o sucesso (Bandura, 1986). Entende-se que, assim como qualquer outra atividade, a repetição tende a proporcionar maior possibilidade perfeição, por exemplo, um estudante de Contabilidade que investe mais tempo resolvendo listas de exercícios de Teoria da Contabilidade tende a ser mais conhecedor de diferentes aplicabilidades que aquele que só resolveu apenas uma lista, ou parte delas. Conclui-se que existe uma relação direta entre maior tempo de estudo e a autorregulação na aprendizagem.

A relação negativa entre escolaridade do pai e autorregulação da aprendizagem foi outro achado dessa pesquisa. O beta não padronizado foi de $-0,063$ e sig. de 0,017 . No estudo exploratório de Castro (2007), em Portugal, foi constatada a existência de uma associação significativa e positiva entre a escolaridade dos pais e o comportamento autorregulatório do estudante, assim como para a percepção de autoeficácia. A literatura de Zimmerman \& Martinez-Pons (1986) e Rosário (1999) indicam que pais mais escolarizados tendem a influenciar positivamente na autorregulação do indivíduo. Uma possível explicação para o achado divergente desse estudo pode estar associado ao perfil econômico dos discentes de Ciências Contábeis serem de baixa a média renda (Iudicíbus, 2012), ou seja, os estudantes se sentiriam mais motivados a uma possibilidade de mudança na sua estrutura econômica e, assim, mais autorregulado nos estudos. Ou seja, a hipótese um (H1) desse estudo pode ser aceita parcialmente, uma vez que nem todas as variáveis demográficas foram estatisticamente significativas com a aprendizagem autorregulada.

Tabela 7

Análise múltipla dos Fatores Acadêmicos

\begin{tabular}{r|ccc|ccc|ccc}
\hline \multicolumn{1}{c|}{ Variáveis } & Coeficiente & Estatística-t & sig. & Coeficiente & Estatística-t & sig. & Coeficiente & Estatística-t & sig. \\
\hline (Constante) & 5,418 & 36,225 & 0,000 & 4,928 & 35,092 & 0,000 & 4,928 & 35,092 & 0,000 \\
Semestre & $-0,019$ & $-1,098$ & 0,273 & $-\mathbf{0 , 0 3 6}$ & $-2,175$ & 0,03 & $-\mathbf{0 , 0 3 6}$ & $-2,175$ & 0,03 \\
Tipo de IES & $-0,034$ & $-0,383$ & 0,701 & $-0,075$ & $-0,912$ & 0,362 & $-0,075$ & $-0,912$ & 0,362 \\
\hline $\mathrm{N}$ & 713 & & & 713 & & & 713 & & \\
$\mathbf{R}^{2}$ & 0,103 & & & 0,008 & & & 0,008 & \\
F (sig.) & 11,688 & & & 2,712 & & & 2,712 & \\
\hline
\end{tabular}

Fonte: Dados da pesquisa (2018). 
A análise da Tabela 7 permite indicar que a variável semestre apresentou um coeficiente beta de - 0,036 e sig. de 0,30, de modo a sugerir uma relação negativa entre o avanço no curso de Ciências Contábeis e o autorregulação na aprendizagem. Ou seja, o avanço no curso não permite que os estudantes se apropriem e desenvolvam mais estratégias de aprendizado autorregulado. A literatura internacional tem indicado que os espaços formais de ensino e aprendizagem promovem a utilização e o desenvolvimento de habilidades da autorregulação da aprendizagem pelos estudantes (Ames, 1992; Abreu, 1991; Almeida, 1993; Rosário, 1999; Castro, 2007). Paradoxalmente, a literatura nacional tem apontado que os alunos de Contabilidade no início do curso são mais autorregulados do que quando avançam (Silva, et al., 2017; Aguiar \& Silva, 2017; Lima Filho, Lima \& Bruni, 2013). Esse cenário permite enfatizar pelo menos três apontamentos e reflexões: primeiro, os estudos realizados internacionalmente, principalmente em Portugal, utilizam de programas de intervenção no aprendizado para desenvolver habilidades cognitivas e metacognitivas nos estudantes, circunstâncias que promovem aumento na capacidade autorregulatórias, uma vez que para Zimmerman \& Martinez-Pons (1986) e Rosário (1999) a aprendizagem das estratégias permitem aplicá-las em múltiplos cenários; segundo, que deve ser estudado o porquê de ocorrer de menos utilização de estratégias autorreguladas à medida que o estudante avança no curso, se por desmotivação, insatisfação, trabalho, entre outros; terceiro, presume-se que possivelmente os professores de Contabilidade possam não estar se utilizando de ações didáticas que promovam o uso dessas estratégias de autorregulação, uma vez que se trata de uma estrutura fortemente técnica.

Foi analisado um terceiro cenário, que pretendeu verificar a relação entre a aprendizagem autorregulada e fatores Sociais e Acadêmicos, com a expectativa de que os dois fatores podem explicar simultaneamente, a Aprendizagem Autorregulada do aluno.Porém, nenhuma outra inferência adicional foi possível estabelecer na análise.

\section{Conclusão}

Este estudo teve como objetivo analisar se fatores demográficos e acadêmicos podem influenciar o uso de estratégias de Aprendizado Autorregulado, elucidando tais aspectos a partir de uma pesquisa realizada com estudantes do curso de Ciências Contábeis, por meio da participação de 713 discentes, adivindos de três IES localizadas na cidade de Salvador, Bahia.

Foi constatado que os alunos utilizam as estratégias de aprendizagem autorregulada com uma frequência superior a 68\%. Dentre as estratégias da autorregulação, as mais utilizadas por discentes de Contabilidade foram a de autoavaliação (E1), a procura de ajuda social (E9), estratégia de estrutura ambiental (E6) e a estratégia de revisão de dados (E10). Zimmerman e Martinz-Pons (1986) assevera que quanto maior for a utilização das estratégias pelos estudantes maior será a autorregulação, que por conseguinte, refletirá no seu desempenho.

O segundo objetivo específico examinou as dimensões das estratégias autorreguladas e constatou-se que, entre as quatorze características identificadas por Zimmerman como frequentemente utilizadas por estudantes autorregulados, neste estudo foram evidenciadas por meio de dois fatores. O primeiro fator, execução, agrupou a "estratégia de estrutura ambiental" (E6), "repetição e memorização" (E8), "procura por ajuda" (E9) e "estratégia de revisão de dados" (E10). E o segundo fator chamado de planejamento e reação, agrupou as estratégias "autoavaliação" (E1), "estabelecimento de objetivos e planejamento" (E3) e "procura de informação" (E4).

O terceiro objetivo foi verificar se fatores demográficos e acadêmicos de estudantes de Contabilidade poderiam influenciar na autorregulação da aprendizagem, que correspondeu às duas hipóteses testadas. Os resultados foram analisados consoante com os fatores "execução" e "planejamento e reação" (agrupados pela análise fatorial) e do índice de aprendizado autorregulado e concluiu a significância com as variáveis gênero, tempo de estudo, idade e escolaridade do pai. Assim, jovens do gênero feminino, que alocam mais tempo aos estudos e que têm pais com menos escolaridade, indicou ter um comportamento mais autorregulado na sua aprendizagem. 
Por meio dos fatores "execução" e "planejamento e reação" e do índice de aprendizado autorregulado, foi indicada relação significativa e negativa apenas para a variável semestre, o que permitiu aceitar parcialmente a hipótese instituída. Nesse ínterim, estudantes de graduação em Contabilidade são mais autorregulados no início do curso do que quando avançam na graduação. Esse achado é alarmante, uma vez que o ambiente universitário deveria prover mecanismos para desenvolver habilidades autorreguladas e, não, as reduzir.

Embora os achados estatísticos tenham, em sua grande parte, apresentado resultados semelhantes para os fatores acadêmicos analisados na SRL no Brasil, esse estudo utilizou técnicas de análise de dados diferentes. Os resultados apontados pelos testes estatísticos permitiram constatar e apresentar outra evidência de característica que influencia a autorregulação da aprendizagem: a relação negativa apenas para a escolaridade do pai. Uma possível explicação para o achado pode estar associada ao perfil econômico dos discentes de Ciências Contábeis serem de baixa a média renda, ou seja, os estudantes se sentiriam mais motivados a uma possibilidade de mudança na sua estrutura econômica e assim mais autorregulado nos estudos. Quanto ao gênero feminino ser mais autorregulado, convergiu com a literatura nacional e contraria a internacional, fato que merecem atenção as possíveis explicações. As variáveis "tempo de estudo" e "trabalho ou estágio" apresentaram relações lógicas de "maior investimento, maior retorno", de modo que quanto mais tempo alocado aos estudos maior a sua autorregulação. Carece explicações, contudo, o fato de estudantes de Contabilidade avançarem no curso e se tornarem menos autorregulados.

Enfatiza-se que não foram identificadas pesquisas que abordassem como o contexto educacional brasileiro se articula para promover a disseminação e promoção sobre a autorregulação da aprendizagem em ambientes universitários. São escassos, também, os estudos que relatem casos de interferências pedagógicas no sentido de promover aspectos metacognitivos e autorreguladas nos estudantes. Assim, indicam-se como lacunas futuras discussões sobre tais aspectos.

\section{Referências}

Abreu, M. V. (1991). Situação actual da avaliação escolar: Alto risco de alienação dos objectivos do sistema educativo. Psychology, 90(1), pp. 94-101.

Aecc. (1990) - Accounting Education Change Commission. Position statement no. one: objectives of education for accountants. Issues In Accounting Education, 5, pp.307-312. doi: 10.12691/jbms-4-1-3

Aguiar, J.H.S. \& Silva, A.C.R. (2015). da. Análise de Características Sociais e Acadêmicas no Aprendizado Autorregulado de Discentes em Contabilidade. Anais.do Congresso Nacional de Administração e Contabilidade, Rio de Janeiro, RJ, Brasil, 6.

Aguiar, J.H.S. \& Silva, A.C.R. (2017). Aprendizado autorregulado em contabilidade: uma análise comparativa entre discentes de modalidade presencial e a distância. Revista Catarinense da Ciência Contábil, Florianópolis, SC, 16(48), pp. 7-23, maio/ago. doi: http://dx.doi.org/10.16930/2237-7662/ rccc.v16n 48.2374

Aicpa. (2000) - American Institute of Certified Public Accountants. The AICPA core competency framework for entry into the accounting profession. doi: 10 1080/0963928031000074486

Almeida, L. (1993). Rentabilizar o ensino-aprendizagem escolar para o sucesso e otreino cognitivo dos alunos. In L. Almeida (Coord.), capacitar a escola parao sucesso, pp. 59-110, V.N. Gaia: Edipsico.

Ames, C. (1992). Classrooms: Goals, structures, and student motivation. Journal of Educational Psychology, 84(3), pp. 261-271. doi: 10.1037/0022-0663.84.3.261

Bandura, A. A. (2008). Teoria social cognitiva na perspectiva da agência”. In: Bandura, A.; Azzi, R.; Polydoro, S. A. J. (orgs.). Teoria Social Cognitiva: conceitos básicos. Porto Alegre, Artmed, pp. 69-96. doi: http://dx.doi.org/10.1590/S0103-166X2009000400016 
(2006). Adolescent development from an agentic perspective. In F. Pajares \& T. C. Urdan (Ed.), Self-Efficacy Beliefs of Adolescents. Greenwich, CT: Information Age Publishing, pp. 1-43.doi: 10.1080/02702710801982159

(1991). Self-regulation of motivation through anticipatory and self- reactive mechanisms. In: Dienstbier, R.A. (Ed.) Perspectives on motivation: Nebraska symposium on motivation. Lincoln, University of Nebraska Press, 38, pp. 69-164.

. (2001). Social Cognitive Theory: An agentic perspective. Annual Reviews Psychologie, 52, pp. 1-26, 2001. doi: 10.1146/annurev.psych.52.1.1

Bandura, A. (1986). Social foundations of thought and action: A social cognitive theory. New Jersey: Prentice-Hall, Inc.

Bandura, A., Caprara G.V., Barbaranelli, C., Gerbino M. \& Pastorelli, C. (2003). Role of affective self-regulatory efficacy in diverse spheres of psychosocial functioning. Child Dev. May-Jun;74(3), pp. 76982. doi:10.1111/1467-8624.00567

Bell, P. D. \& Akroyd, D. (2006) Can factors related to self-regulated learning predict learning achievement in undergraduate asynchronous Web-based courses.ÉInternational Journal of Instructional Technol- ogy and Distance Learning. 3(10), pp. 5-16.

Boekaerts, M. (1999) Self-regulated learning: Where we are today. International Journal of Educational Research, 31, pp. 445 e 457. doi: 10.1016/S0883-0355(99)00014-2

Boekaerts, M. \& Cascallar. E. (2006). How far have we moved toward the integration of theory and practice in self-regulation?Educational Psychology Review, 18(3), pp. 199-210. doi: https://doi.org/10.1007/ s10648-006-9013-4

Bruni, A. L. (2012). Spss: Guia Prático Para Pesquisadores. São Paulo, Editora Atlas.

Castro, M. (2007). Processos de auto-regulação da aprendizagem: Impacto de variáveis académicas e sociais. Dissertação de mestrado, Instituto de Educação e Psicologia, Universidade do Minho, Portugal.

Catell, R. R. (1966). The scree test for number of factors, Multivariate Behavioral Research, 1, pp. 245-276.

Corno, L. (2001). Volitional aspects of self-regulated learning. In B.J. Zimmerman \& D.H. Schunk (Eds.), Self-regulated learning and academic achievement: Theoretical perspectives (2nd edn., pp. 191226). Mahwah, NJ: Lawrence Erlbaum. doi: https://doi.org/10.4324/9781410601032-10

Corrêa-Vieira, D., Bragagnolo-Frison, L. \& Veiga-Simão, A. (2015). Aprendizagem Autorregulada: estratégias de compreensão leitora. Revista De Estudios E Investigación En Psicología Y Educación, 1, pp. 072-077. https://doi.org/10.17979/reipe.2015.0.01.392

Dancey, Christine \& Reidy, John. (2006), Estatística Sem Matemática para Psicologia: Usando SPSS para Windows. Porto Alegre, Artmed.

Efklides, A. (2011). Interactions of metacognition with motivation and affect in self-regulated learning: the MASRL model. Educational Psychologist, 46, pp. 6-25. doi: 10.1080/00461520.2011.538645

Ficher, C. \& Miller, G. (2000). Learning strategies for distance education students. Journal of Agricultural Education, 41(1), pp. 60-68.

Fischer, T., Davel, E. \& Vergara, S. (2006). Razão e sensibilidade no ensino de administração: a literatura como recurso estético. Encontro Nacional dos Programas de Pós-Graduação em Administração, Salvador, BA, Brasil, 30 .

Frison, L.M.B. (2016). Monitoria: uma modalidade de ensino que potencializa a aprendizagem colaborativa e autorregulada. Pro-Posições, 27(1), pp. 133-153, jan./abr. doi: http://dx.doi.org/10.1590/01037307201607908 
Gil, A. C. (2007). Como elaborar projetos de pesquisa. $4^{\text {a }}$. ed. São Paulo: Atlas.

Hair, J., Black, W., Babin, B., Anderson, R. \& Tatham, R. (2006). Multivariate data analysis (6th ed.). Uppersaddle River, N.J.: Pearson Prentice Hall.

Herndon, J. S. \& Bembenutty, H. (2017). Self-regulation of learning and performance among students enrolled in a disciplinary alternative school. Personality and Individual Differences, pp. 104 266-271. doi: http://dx.doi.org/10.1016/j.paid.2016.08.027

Hoffmann, R (2006). Análise de regressão: uma introdução à econometria. HUCITEC: São Paulo.

Iudícibus, S.(2012). Teoria da contabilidade: evolução e tendências. Revista de Contabilidade do Mestrado em Ciências Contábeis da UERJ, 17(2), pp. 5-13.

Lima Filho, R. N., Lima, G. A. S. F. \& Bruni, A. L. (2015). Aprendizagem autorregulada em Contabilidade: diagnósticos, dimensões e explicações. Brazilian Business Review, 12(1), pp. 38-56. doi: http:// dx.doi.org/10.15728/bbr.2015.12.1.2

Menezes, I. G. (2006). Escala de Intenções Comportamentais de Comprometimento Organizacional (Eicco): Concepção, Desenvolvimento, Validação E Padronização. Dissertação (Mestrado em Psicologia). PPGPSI. Universidade Federal da Bahia. Salvador, BA, Brasil.

Miloca, Simone A. (2002). Aplicacão da Teoria de Correlação Canõnica e mêtodo PRO-METHEE num problema de qualidade industrial do trigo, Curitiba, UFPR.

Netemeyer, R. G.; Bearden, W. O.; Sharma, S. (2003). Scaling procedures: Issues and Applications. London, Sage Publications.

Krauss, J.A.; Silva, T.B.J. \& Zonatto, V.C.S (2017). Evidências dos efeitos das estratégias metacognitivas de aprendizagem autorregulada na formação de inconsciente coletivo no conhecimento de contabilidade de custos. Anais do Congresso Anpcont, Belo Horizonte, MG, Brasil, 11.

Polydoro, S.A.J. \& Azzi, R. G. (2008). “Autorregulação: aspectos introdutórios”. In: Bandura, A.; Azzi, R.; Polydoro, S. A. J. (orgs.). Teoria Social Cognitiva: conceitos básicos. Porto Alegre, Editora Artmed, pp. 149-164

Ribeiro, C (2003). Metacognição: um apoio ao processo de aprendizagem. Psicologia: Reflexão e Crítica, 16(1), pp. 109-116. doi: http://dx.doi.org/10.1590/S0102-79722003000100011

Rosário, P. (1999). Variáveis cognitivo-motivacionais na aprendizagem: As abordagens ao estudo em alunos do Ensino Secundário. Tese de doutorado, Universidade do Minho, Braga, Portugal.

Rosário, P. (2002) (Des)venturas do TESTAS. Estórias sobre o estudar, histórias para estudar. Narrativas auto-regulatórias na sala de aula. Porto: Porto Editora.

(2001). Diferenças processuais na aprendizagem: Avaliação alternativa das estratégias de auto-regulação da aprendizagem. Psicologia, Educação e Cultura, 5(1), pp. 87-102. doi: https://doi. org/10.17524/repec.v11i1.1412

Rosário, P. \& Almeida, L. (2005). Leituras construtivistas da aprendizagem. Em: G. Miranda; S. Bahia (Eds.). Psicologia da educação: temas de desenvolvimento, aprendizagem e ensino, pp. 141-165. Lisboa: Relógio D’água Editores. . (2004). Estudar o estudar: As (Des)venturas do Testas. Porto: Porto Editora,2004

Rosário, P. \& Polydoro, S.A.J. (2012). Capitanear o aprender: promoção da autorregulação da aprendizagem no contexto escolar. São Paulo, Casa dos Psicólogos.

Rosário, P.; Mourão, S.S.R. \& Chaleta, L.G.E. (2005). Trabalho de casa, tarefas escolares, auto-regulação e envolvimento parental. Psicologia em Estudo, Maringá, 10(3), pp. 343-351. doi: http://dx.doi. org/10.1590/S1413-73722005000300002 
Sampaio, R., Polydoro, S. \& Rosário, P (2012). Autorregulação da aprendizagem e a procrastinação acadêmica em estudantes universitários. Cadernos de Educação, (42), pp. 119-142. http://dx.doi. org/10.15210/caduc.v0i42.2151.

Schunk, D. H. (2005). Self-regulated learning: The educational legacy of Paul R. Pintrich. Educational Psychologist, 40, pp. 85-94. Retrieved from http://libres.uncg.edu/ir/uncg/f/D_Schunk_Self_2005.pdf

Schunk, D. H., Pintrich, P. R. \& Meece, J. L. (2008). Motivation in education: theory, research, and applications (3rd ed.). Upper Saddle River, N.J.: Pearson/Merrill Prentice Hall.

Schunk, D. H. \& Zimmerman, B. (1997). Social origins of self-regulatory competence. Educational Psychologist Review, 32(4), pp. 195-208. doi: https://doi.org/10.1207/s15326985ep3204_1

Schunk, D.H. \& Zimmerman, B.J. (2003). Self-regulation and learning. In Reynolds, W.M.\& Miller, G.E. (Eds.), Handbook of psychology Volume 7 Educational Psychology (pp.59-78). John Wiley \& Sons, Inc: New Jersey.

Shimada, A. T., Chiusoli, C. L. \& Messetti, A. V. L. (2010). Análise fatorial: avaliação de estabelecimentos alimentícios. Anais Seminários em Administração (Semead), Universidade de São Paulo, São Paulo, SP, Brasil, 13.

Shuell, T. (1986). Cognitive Conceptions of Learning. Review of Educational Research, 56(4), pp. 411-436. doi: https://doi.org/10.3102/00346543056004411

Silva, A. (2004). A auto-regulação na aprendizagem. A demarcação de um campo de estudo e de intervenção. In A. Silva, A. Duarte, I. Sá \& A. M. Veiga Simão, Aprendizagem AutoRegulada pelo Estudante. Perspectivas psicológicas e educacionais, pp.17-39, Porto: Porto Editora.

Silva Júnior, S. D. \& Costa, F. J. (2014). Mensuração e Escalas de Verificação: uma Análise Comparativa das Escalas de Likert e Phrase Completion. PMKT - Revista Brasileira de Pesquisas de Marketing, Opinião e Mídia, São Paulo, Brasil, 15, pp. 1-16,

Silva, J. \& Veiga Simão, A. M. (2016). Entrevista com tarefa na identificação de processos na aprendizagem autorregulada. Psicologia Escolar e Educacional, São Paulo, SP, 20(1), pp. 89-100. DOI: 10.1590/2175$3539 / 2015 / 0201932$

Silva, T., Lay, L. A., Hein, N., Biavatti, V. \& Zonatto, V. (2017). As Estratégias de Aprendizagem Autorregulada (SRL) no Ensino EAD de Contabilidade. Revista De Educação E Pesquisa Em Contabilidade (REPeC), 11(1). https://doi.org/10.17524/repec.v11i1.1412

Veiga Simão, A.M. da. \& Frison, L.M.B. (2013). Autorregulação da aprendizagem: abordagens teóricas e desafios para as práticas em contextos educativos. Cadernos de Educação, FaE/PPGE/UFPel, Pelotas, (45) pp. 2 - 20, maio/agosto. doi: 10.24220/2318-0870v21n1a2992

Zimmerman, B. (1989). A social cognitive view of self-regulated academic learning. Journal of Educational Psychology, 81(3), 329-339. doi: http://dx.doi.org/10.1037/0022-0663.81.3.329

. (2002). Becoming a self-regulated learner: An overview. Theory into Practice. 41(2), pp. 64-70. doi: https://doi.org/10.1207/s15430421tip4102_2

Zimmerman, B. (1998). Developing self-fulfilling cycles of academic regulation: An analysis of exemplary instructional models. In D. Schunk \& B. Zimmerman (Eds), Self-regulated learning: From teaching to self-reflective practice, pp. 1-19, New York: Guilford Publications.

Zimmerman, B, J. (2002). Becoming a self-regulated learner: An overview. Theory Into Practice, 41, 2, pp. 64-70. Retrieved from http://commonsenseatheism.com/wp-content/uploads/2011/02/Zimmerman-Becoming-a-self-regulated-learner.pdf

Zimmerman, B. J.; Bonner, S.; Kovach, R. (1996). Developing self-regulated learners; Beyond achievement to self-efficacy. Washington, DC: American Psychological Association. 
Zimmerman, B. J. \& Cleary, T. J. (2006). Adolescents' development of personal agency: The role of selfefficacy beliefs and self-regulatory skill. In F. Pajres, \& T. Urdan (Eds.), Self-efficacy beliefs of adolescence Mahwah, NJ7 Information Age Publishing, pp. 45 - 69.

Zimmerman, B. J. (2000). Attaining self-regulation: A social-cognitive perspective. In: M. Boekaerts, M.; Pintrich, P. \& Zeidner, M. (eds.). Self-regulation: Theory, research, and applications. Orlando, FL7 Academic Press, pp.13-39. doi: http://dx.doi.org/10.1016/B978-012109890-2/50031-7

Zimmerman, B. J. (2000a). Self-efficacy: an essential motive to learn. Contemporary Educational Psychology, 25, pp. 82-91.

Zimmerman, B. J. (2000b). Attaining Self-Regulation: A Social Cognitive Perspective. In M. Boekaerts, P. R. Pintrich, \& M. Zeidner (Eds.), Handbook of Self-Regulation, pp. 13-39. San Diego, CA: Academic Press. http://dx.doi.org/10.1016/B978-012109890-2/50031-7

Zimmerman, B. J. \& Martinez-Pons, M. (1986). Development of a Structured Interview for Assessing Student Use of Self-Regulated Learning Strategies. American Educational Research Journal, Winter, Washington, 23(4), pp. 614-628. doi: 10.3102/00028312023004614

Zimmerman, B. J. \& Schunk, D. H. (Eds.). (1989). Springer series in cognitive development. Self-regulated learning and academic achievement: Theory, research, and practice. New York, NY, US: Springer-Verlag Publishing. http://dx.doi.org/10.1007/978-1-4612-3618-4

Zimmerman, B.J. \& Schunk, D.H. (2004). Self regulating intellectual processes and outcomes: Social cognitive perspective. In D.Y. Dai, \& R.J. Stenberg (Eds.), Motivation, emotion, and cognition: Integrative perspectives on intellectual functioning and development, pp. 323-350. Mahwah, NJ: Erlbaum. 